\title{
Improvement of Si Field Emission Sensors Fabrication Technology by Carbon Nanotubes
}

\author{
Michel O.S. Dantas ${ }^{1}$, Elisabete Galeazzo ${ }^{2}$, Henrique E. M. Peres ${ }^{2}$, Francisco J. Ramirez-Fernandez ${ }^{2}$ \\ ${ }^{1}$ Centro de Engenharia, Modelagem e Ciências Sociais Aplicadas (CECS) - UFABC \\ Rua Santa Adélia, 166. Santo André - SP - Brazil \\ ${ }^{2}$ Laboratório de Microeletrônica (LME) - Escola Politécnica - Universidade de São Paulo - EPUSP \\ Av. Prof. Luciano Gualberto, trav. 3, 158. São Paulo - SP - Brazil \\ Corresponding e-mail address: michel.dantas@ufabc.edu.br
}

\begin{abstract}
Field Emission (FE) devices were successfully improved by the association of silicon (Si) microtips and Multi-Walled Carbon Nanotubes (MWCNTs). MWCNTs were deposited over Si microtips by Electrophoretic Deposition (EPD), and improvements up to $80 \%$ in electron emission characteristics were reported, being suitable for the development of gas ionization sensors based on FE devices.
\end{abstract}

Key words: Field Emission, Carbon Nanotubes, Electrophoretic Deposition, gas ionization sensors

\section{Introduction}

Studies involving the feasibility of applying carbon nanotubes (CNTs) in the development of miniaturized ionization gas sensors based on the field emission effect have recently been carried out, and the results are very promising [1]. CNTs are interesting material for field emission applications due to their remarkable caracteristics as high aspect ratio (height to diameter ratio), sharp tip structure, good electric conductivity, and high mechanical and chemical stability [2].

In CNT field emitters, strong electric fields are concentrated at CNTs tips, consequently requiring lower operating voltages. On the other hand, silicon $(\mathrm{Si})$ is also an interesting material for fabrication of field emitters. Although of their field emission properties are not so attractive (or superior) as compared to other materials, silicon has the advantage of being a suitable material for the manufacture of signal conditioning circuits [2].

Besides, Si field emission efficiency can be improved by the coating of its surface with several materials, which provides, among other characteristics, protection of microtips against sputtering by gaseous substances accelerated against them.

Thus, our proposal is to combine the advantage of fabricating field emission devices using $\mathrm{Si}$ microtips arrays with the excellent properties of CNTs. By applying CNTs on top of $\mathrm{Si}$ microstructures we hope this procedure can increase the efficiency and stability of electron emission of the sensors devices.

\section{Experimental Procedure}

Three samples with Si microtips were obtained by the well-established HI-PS technique [4]. Next, the electrophoretic deposition (EPD) method was employed to cover Si microtips with multi-walled carbon nanotubes (MWCNTs), as detailed as follows.

\section{MWCNT Electrophoretic Deposition}

Various methods have been developed and reported for depositing CNTs on surfaces. In particular, EPD is a cost-effective method commonly used in the processing of ceramics, coating and composite materials, as well as electrode coatings. EPD is efficient and useful for depositing thin and thick CNT films on conductive surfaces [5]. However, to conduct the process of electrophoresis, carbon nanotubes must be dispersed in aqueous solution and chemically functionalized.

In this work, MWCNTs were functionalized as described previously in [6] and, before the EPD process, the solution was sonicated for 2 hours. Then, MWCNTs dispersed in the solvent were deposited over three samples (A, B and C) exploring the variation of electrophoretic current as follows: Sample A ( $\left.\mathrm{I}_{\mathrm{EPD}}=0.2 \mathrm{~mA}\right)$, Sample B $\left(l_{E P D}=0.5 \mathrm{~mA}\right)$ and Sample $C\left(I_{E P D}=20 \mathrm{~mA}\right)$. The EPD process time for all samples was 4 minutes. The aim of this procedure was to find the best CNTs deposition condition over arrays of Si microtips. All EPD processes were performed with a small electrolytic cell, filled with $1 \mathrm{~cm}^{3}$ of MWCNT solution, and the distance between the electrodes was kept constant $(7.6 \mathrm{~mm})$, as represented in Figure 1 . 
EPD process was carried out with a Source and Measurement Unit (SMU), model 236 (Keithley Instruments) controlled by Virtual Instrumentation with LabVIEW $^{\circledR}$ Software (National Instruments). After EPD, samples were left to dry in air.

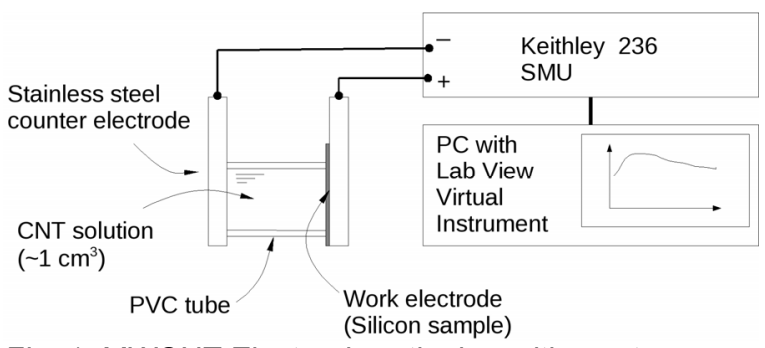

Fig. 1: MWCNT Electrophoretic deposition setup.

\section{Morphological and Electrical Characterization}

The Si microtips were characterized morphologically by optical and electron microscopy before and after electrophoretic deposition of WMCNTs. A NOVA 400 NanoSEM (FEI Company ${ }^{\mathrm{TM}}$ ) scanning electron microscope (SEM) was used, operating at $5 \mathrm{keV}$ to avoid damages on deposited CNTs. In order to measure the field emission current of electrons from the microtips, the samples were mounted in a high vacuum chamber with pressure about $2 \times 10^{-6}$ Torr to avoid influences of residual gases ionization on the emitted current. As illustrated in Figure 2, the Si sample is fixed on a cathode connected to ground through a Programmable Electrometer model 617 (Keithley Instruments), while a planar stainless steel anode, which has a circular area around $0.8 \mathrm{~cm}^{2}$, is connected to the positive terminal of a High Voltage Supply model 248 (Keithley Instruments). A series protection resistor is included in order to protect instruments against current peaks due to undesired sparks into the chamber.

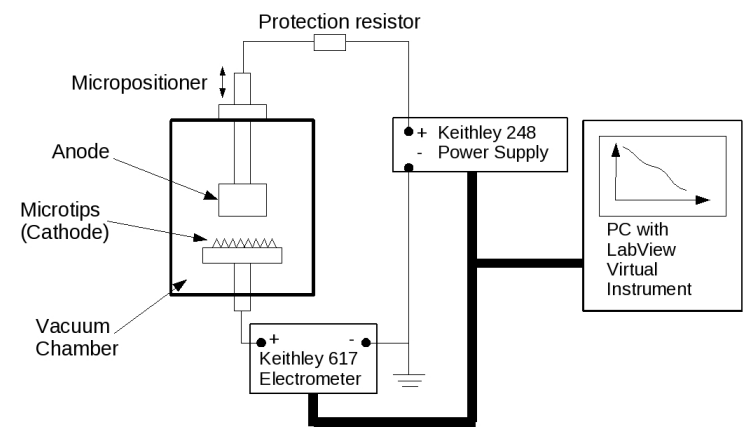

Fig. 2: Electrical system setup for FE devices characterization.

Another virtual instrument software developed in LabVIEW ${ }^{\circledR}$ controls the high voltage source and stores the measured current values, graphically showing the results during the tests (I-V and FN curves). In this setup, the distance (d) between the sample and the anode can be adjusted externally by a micropositioner coupled to the anode, in such a way that electrical characterization can be made by varying the distance between electrodes, but keeping the vacuum environment conditions. Furthermore, the anode structure has a selfaligned fixation system, in order to compensate mechanical misalignment.

In this work, electrical characterizations were carried out for electrodes distances from 80 to $200 \mu \mathrm{m}$ for Si microtips samples without MWCNTs, and from 100 to $500 \mu \mathrm{m}$ for Si microtips samples coated with them. For each distance, a voltage ramp was applied limiting the maximum emission current to about $20 \mu \mathrm{A}$, in order to avoid eventual spikes and CNT burnout. From experimental measures made with this apparatus, three kinds of curves, obtained for several separation distances $d$ between electrodes, were made: I-V, FN-plot and $V-d$. The $I-V$ curves were used to verify if the experimental curve aspect is in accordance with the expected from the Fowler-Nordheim theory. Based on their model, the electron emission current density is governed by the following exponential-like behavior equation [7]:

$J=\frac{c_{1} \times(\beta \times E)^{2}}{\phi} \times \exp \left(-\frac{c_{2} \times \phi^{3 / 2}}{\beta \times E}\right)$

where $c_{1}$ and $c_{2}$ are constants, $E$ is the macroscopic applied electrical field $(E=V / d), \phi$ is the material work function, $\beta$ is called "field enhancement factor", which represent improvement in the emission current obtained by electrode optimizations (microtips structure, for example) [7]. From the $l-V$ curves, voltages required to provide $12 \mu \mathrm{A}$ of emission current are extracted, and the results are used to build $V$ - $d$ curves, which are applied for extracting macroscopic electrical field $E$.

Finally, the FN-plot was applied to extract factor $\beta$, which can be used as quality comparison parameter among samples. $\beta$ is obtained by the analysis of the FN-plot slope $(\alpha)$ [7]:

$\beta=\frac{6.53 \times 10^{9} \times d \times \phi^{3 / 2}}{\alpha}$

\section{Results}

Si microtips coating with MWCNTs were obtained with a variation of two order of magnitude of electrophoretic current (0.2 to $20 \mathrm{~mA}$ ). From the images shown in Figure 3, 
poor or excessive deposition of CNT over arrays can be observed (samples $A$ and $C$, respectively). These results were dependent on the EPD current applied. A selective MWCNTs deposition on the tips array area was achieved in Sample $B$ by applying $0.5 \mathrm{~mA}$.
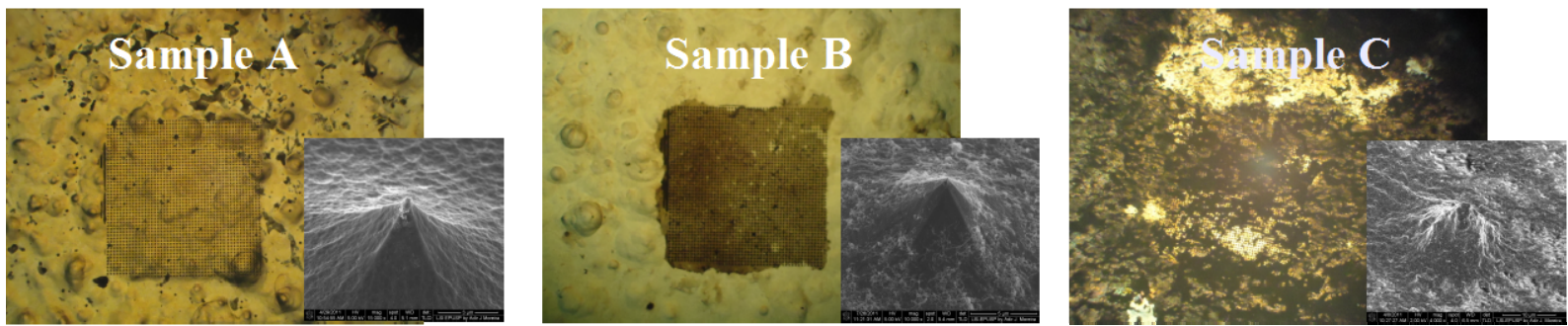

Fig. 3: Images of Optical Microscopy and SEM (in detachment) after CNTS EPD on samples A ( $I_{E P D}=0.2 \mathrm{~mA}$, poor tips coverage), $B\left(I_{E P D}=0.5 \mathrm{~mA}\right.$, preferential coverage on tips array area) and $C\left(I_{E P D}=20 \mathrm{~mA}\right.$, excessive coverage).
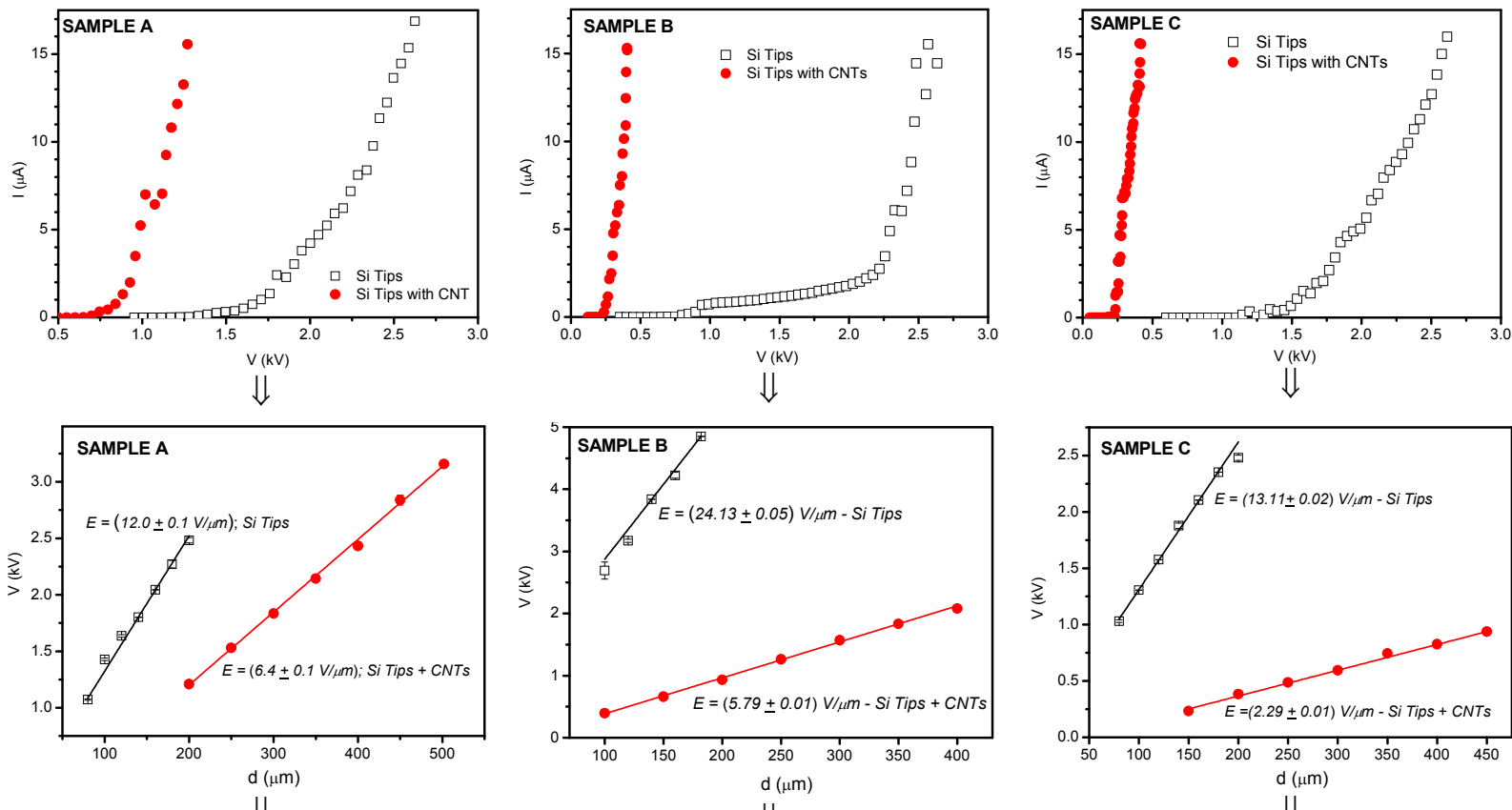

$\Downarrow$
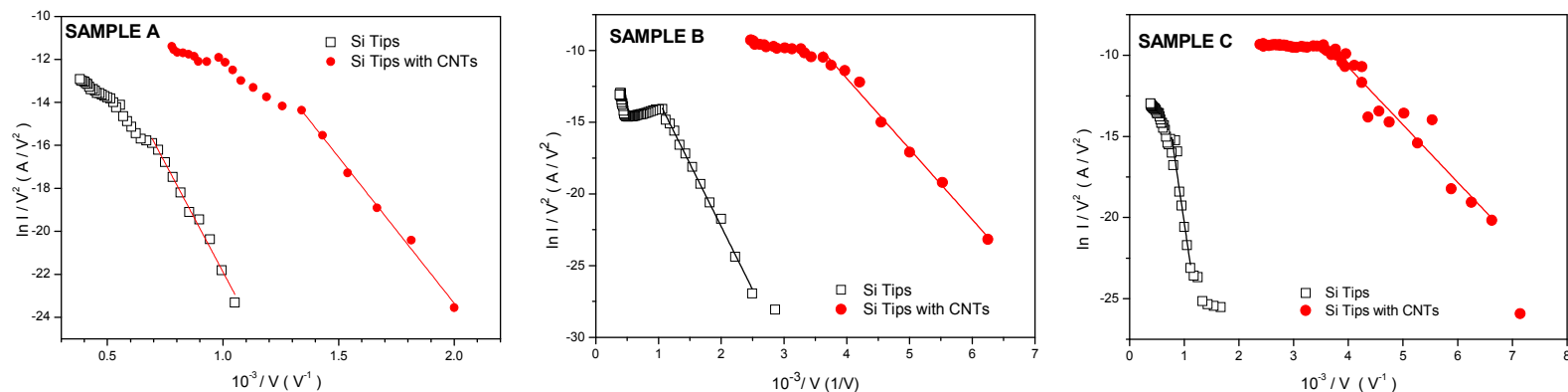

Fig. 4: I-V (top), V-d (middle) and FN (bottom) curves before and after CNTs deposition for samples A, B and C. A substantial decrease of applied potential and macroscopic electrical field is observed for all samples with CNTs, demonstrating the improvement of emission characteristics after CNTs electrophoretic deposition.

All samples showed improvement in emission characteristics after MWCNTs deposition: Figure 4-top shows $I-V$ curves with separation distance $d$ between electrodes of $100 \mu \mathrm{m}$ (sample B) and $200 \mu \mathrm{m}$ (sample A and C), where pronounced reductions of the applied voltages for the same levels of emission currents can be verified. As described in the
Experimental Procedure section, $V$ - $d$ curves (Figure 4-middle) are traced from $I-V$ curves data, for $12 \mu \mathrm{A}$. Thus, one can extract macroscopic electric fields $E$ for each condition. Such analysis quantifies the reduction in electrical field for samples deposited with CNT. It is important to explain that lower $E$ values indicate better emission characteristics, or 
rather, less energy to promote electron emission, and CNTs considerably reduce this parameter, as expected. FN-plots (Figure 4bottom), obtained from $I-V$ data, show changing of slope $\alpha$ for all samples after CNTs deposition indicating, consequently, different $\beta$ values for each condition (with and without CNTs coating). Figure 5 illustrates $\beta$ values extracted from FNplots, which show increase for all samples after CNTs coating. This result is also in accordance with the EPD process parameters and morphological analysis shown in Figure 3: samples obtained with higher EPD current show more density of CNTs per area, raising the $\beta$ value.

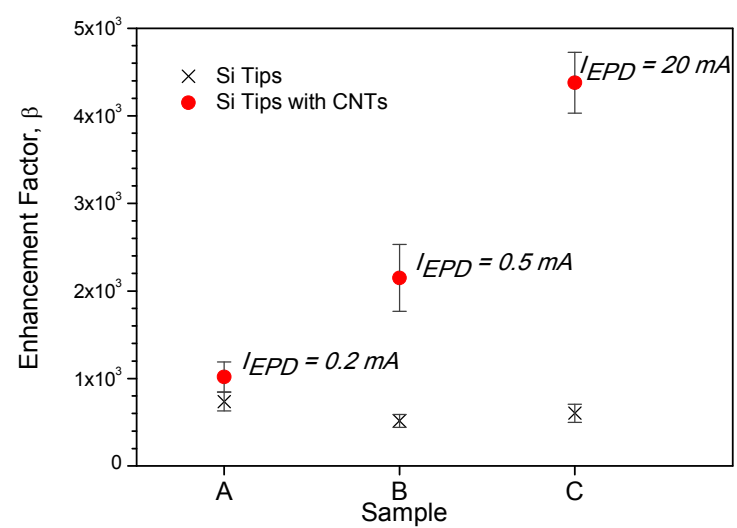

Fig. 5: "Field Enhancement factor" $\beta$ of the samples before (black) and after (red) CNT deposition.

\section{Discussion}

Since, in practical terms, it is impossible to measure $\phi$ and $\beta$ from the same graphic, an estimation of the work function $\phi$ is made to apply equation (2); thus, a considerable imprecision of $\beta$ value can occur. A more precise comparison parameter among samples is the macroscopic electrical field $E$, which can be obtained from $V$ - $d$ curves. The experimental apparatus developed in this work permits obtaining $V$ and $d$ values precisely for a fixed emission current, the relationship of which is very linear, allowing the extraction of $E$ from the slope of the curves easily. In spite of the improvements achieved, some considerations must be made: sample A shows decrease in $E$ by about $47 \%$, which can be related to the poor coverage of microtips by CNTs; samples B and $C$ show incredible decrease of $E$ by about $80 \%$, but it is important to note that in B the CNTs are concentrated only at the region of tips array (as seen in Figure 3), thus, the effectiveness of emission points can be considered higher than in $\mathrm{C}$, the deposition of which occurred not only in the array region, but in all the area exposed to EPD process.

\section{Conclusion}

Si FE devices coated with CNTs showed improvement in their emission characteristics. For the three devices analyzed, the results obtained show that sample B has better electrical and morphological aspects for the development of gas sensors based on field emission, since an enhanced emission occurs in a very well defined area. Next steps include the evaluation of devices such as ionization source for several gases towards the development of integrated gas sensor systems.

\section{Acknowledgements}

We would like to thank LSI at EPUSP for the SEM characterization, N. Peixoto for the supply of the functionalized CNTs chemical solution, C. Mendes, A. Ribeiro, M.C. Moraes for the experimental support, Fapesp (2009/53359-0) for the financial support and CNPq for the scholarship.

\section{References}

[1] H. Liu, H. Ma, W. Zhou, W. Liu, Z. Jie, X. Li, Synthesis and gas sensing characteristic based on metal oxide modification multi wall carbon nanotubes composites, Applied Surface Science 258, 1991-1994 (2012); DOI: 10.1016/j.apsusc.2011.05.081

[2] J.M. Bonard M. Croci, C. Klinke, R. Kurt1, O. Noury, N. Weiss, Carbon nanotube films as electron field emitters, Carbon 40, 1715-1728 (2002); DOI:10.1016/S0008-6223(02)00011-8

[3] S.J. Hong, S.J. Kwon, E.S. Cho, Field emission properties of silicon field emitter arrays with volcano-shaped gate structure, Current Applied Physics 9, 967-971 (2009);

DOI:10.1016/j.cap.2008.09.012

[4] M.O.S. Dantas, E. Galeazzo, H.E.M. Peres, M.M. Kopelvski, F.J. Ramirez-Fernandez, Silicon FieldEmission Devices Fabricated Using the Hydrogen Implantation Porous Silicon (HI-PS) Micromachining Technique, Journal of Microelectromechanical Systems 17, 1263-1269 (2008); DOI:10.1109/JMEMS.2008.927743

[5] A. Boccaccini, J. Cho, J. Roether, B. Thomas, E. Minay, M. Shaffer, Electrophoretic deposition of carbon nanotubes, Carbon 44, 3149 (2006); DOI: 10.1016/j.carbon.2006.06.021

[6] S. Minnikanti, P. Skeath, N. Peixoto, Electrochemical characterization of multi-walled carbon nanotubes coated electrodes for biological applications, Carbon 47, 884-893 (2009); DOI: 10.1016/j.carbon.2008.11.045

[7] J.M. Bonard, M. Croci, I. Arfaoui, O. Noury, D. Sarangi, A. Châtelain, Can we reliably estimate the emission field and field enhancement factor of carbon nanotube film field emitters?, Diamond and Related Materials 11, 763-768 (2002); DOI: 10.1016/S0925-9635(01)00541-6 